\title{
Invaginación sigmoidea como presentación clínica de schwannoma de colon. Caso pediátrico
} Sigmoid intussusception as a clinical presentation of colonic schwannoma. Pediatric case

\author{
Lic. Yurema González Ruiz', Lic. Alvira Reyes Delgado , Lic. Cristina Gutiérrez Alonso ${ }^{b}$, Lic. José I. Franco Rubio \\ y Lic. Marina González Herrero. ${ }^{a}$
}

\section{RESUMEN}

Los schwannomas son tumores que se originan de las células de Schwann de los nervios periféricos. Su localización en el colon es extremadamente rara y no se han descrito, en la literatura, casos en niños. Por lo general, estos tumores son asintomáticos, pero pueden producir síntomas inespecíficos y gastrointestinales. La presentación comoinvaginaciónintestinal es muy infrecuente. Se expone el caso clínico de una paciente de 11 años de edad con un schwannoma de colon manifestado clínicamente como invaginación intestinal de sigma con dolor abdominal, rectorragia y estreñimiento de 10 días de evolución, que precisó resección intestinal con márgenes quirúrgicos libres, con buena evolución posoperatoria.

Palabras clave: schwannoma, colon, neoplasias gastrointestinales, invaginación, sigmoide.

\begin{abstract}
Schwannomas are tumours originating in the Schwann cells of the peripheral nerves. The appearance in the colon is extremely rare, and there are no known cases in the literature of schwannomas of the colon and rectum described in children. These tumours are usually asymptomatic, but can produce non-specific and gastrointestinal symptoms. The clinical presentation as intestinal intussusception is infrequent. We present a case of an 11-year-old girl with sigmoid swchannoma presented clinically as a colonic intussusception with abdominal pain, rectal bleeding and constipation of 10 days. An intestinal resection with free surgical margins was performed.

Key words: schwannoma, colon, gastrointestinal neoplasms, sigmoid, intussusception.
\end{abstract}

http: / / dx.doi.org/10.5546/ aap.2019.e68

Cómo citar: González Ruiz Y, Reyes Delgado A, Gutiérrez Alonso C, Franco Rubio JI, et al. Invaginación sigmoidea como presentación clínica de schwannoma de colon. Caso pediátrico. Arch Argent Pediatr 2019;117(1):e68-e71.

a. Servicio de Cirugía Pediátrica, Hospital Universitario Miguel Servet, Zaragoza, España.

b. Servicio de Radiología y Radiodiagnóstico, Hospital Universitario Miguel Servet, Zaragoza, España.

c. Servicio de Anatomía Patológica, Hospital Universitario Miguel Servet, Zaragoza, España.

Correspondencia:

Lic. Yurema González Ruiz: yuremagr@gmail.com

Financiamiento: Ninguno.

Conflicto de intereses: Ninguno que declarar.

Recibido: 29-5-2018

Aceptado: 1-10-2018

\section{INTRODUCCIÓN}

Los schwannomas son tumores infrecuentes que se originan de las células de Schwann de los nervios periféricos, que representan, únicamente, el 2-6\% de todos los tumores mesenquimales y el $0,1 \%$ de los tumores benignos del tracto gastrointestinal. ${ }^{1,2}$ La incidencia es similar tanto en hombres como en mujeres y tienden a aparecer a partir de la sexta década de vida, aunque pueden surgir a cualquier edad., Estos tumores pueden presentarse a lo largo de todo el cuerpo, pero se localizan, más frecuentemente, en la cabeza, el cuello y las extremidades superiores e inferiores. En el tracto gastrointestinal, aparecen con mayor incidencia en el estómago (el $83 \%$ ) y en el intestino delgado (el $12 \%) .{ }^{5}$ La presentación colónica es rara y, cuando aparecen, suelen afectar, en primer lugar, a ciego, seguido de sigma, rectosigma, colon transverso, colon descendente y recto. ${ }^{1,2}$ Los schwannomas de colon y recto se asocian, generalmente, con neurofibromatosis sistémica, como la enfermedad de Von Recklinghausen. ${ }^{6}$ No existen casos descritos en la literatura de schwannomas de colon y recto en niños. Se presenta el caso clínico de una paciente de 11 años de edad con un schwannoma sigmoideo presentado clínicamente como una invaginación intestinal de sigma; ambas patologías son muy raras.

\section{CASO CLÍNICO}

Niña de 11 años que acudió al Servicio de Urgencias de nuestro Hospital por dolor abdominal, estreñimiento y rectorragia de 10 días de evolución. La paciente permanecía afebril y hemodinámicamente estable. Al momento de la exploración física, presentaba distensión abdominal y defensa abdominal en el hipogastrio, $y$, con el tacto rectal, se palpó una masa fibroelástica, redondeada, que prolapsaba por el recto. La analítica mostró leucocitos $13900 / \mathrm{mm}^{3}$, hemoglobina de $13,8 \mathrm{~g} / \mathrm{dl}$ y marcadores tumorales negativos (antígeno carbohidrato-carbohydrate antigen; CA-19,9, 
por sus siglas en inglés-: 10,9 U/ml; antígeno carcinoembriónico -carcinoembryonic antigen; $C E A$, por sus siglas en inglés-: $1 \mathrm{ng} / \mathrm{ml})$. En la radiografía abdominal, existía una dilatación del intestino delgado y del colon, sin evidencia de gas distal en el recto. Tanto en el estudio ecográfico como en la tomografía computada (TC), se evidenció una imagen compatible con invaginación sigmoidea. En la TC, se apreciaba, en el extremo de la invaginación, un nódulo intraluminal, redondeado e hipovascular (Figura 1). Se realizaron un pneumoenema y un hidroenema, que no fueron efectivos. Antes de la intervención, se realizó una colonoscopía, que identificó una tumoración con mucosa isquémica a $10 \mathrm{~cm}$ del margen anal. No fue posible el paso del colonoscopio posterior a la masa, que alcanzó un fondo de saco ciego. En la cirugía, se observó una invaginación de colon sigmoide de $15 \mathrm{~cm}$ de longitud. Se consiguió la desinvaginación completa, y se encontró, como cabeza de la invaginación, una tumoración redondeada intraluminal, firme, grisácea, de $4 \times 3 \times 3 \mathrm{~cm}$, con ulceración de la mucosa (Figura 2). Se realizó la resección del colon sigmoide afecto con márgenes quirúrgicos de $1,5 \mathrm{~cm}$ y anastomosis términoterminal. La paciente evolucionó favorablemente y fue dada de alta el séptimo día posoperatorio, sin precisar ningún tratamiento neoadyuvante.

La histopatología del segmento de sigma resecado confirmó una neoplasia sólida intramural, bien delimitada de $4 \times 3,2 \times 3,2 \mathrm{~cm}$ con márgenes quirúrgicos libres de lesión, constituida por una proliferación de células de morfología fusiforme dispuestas en haces cortos entrecruzados, con formación

Figura 1. Pruebas de imagen

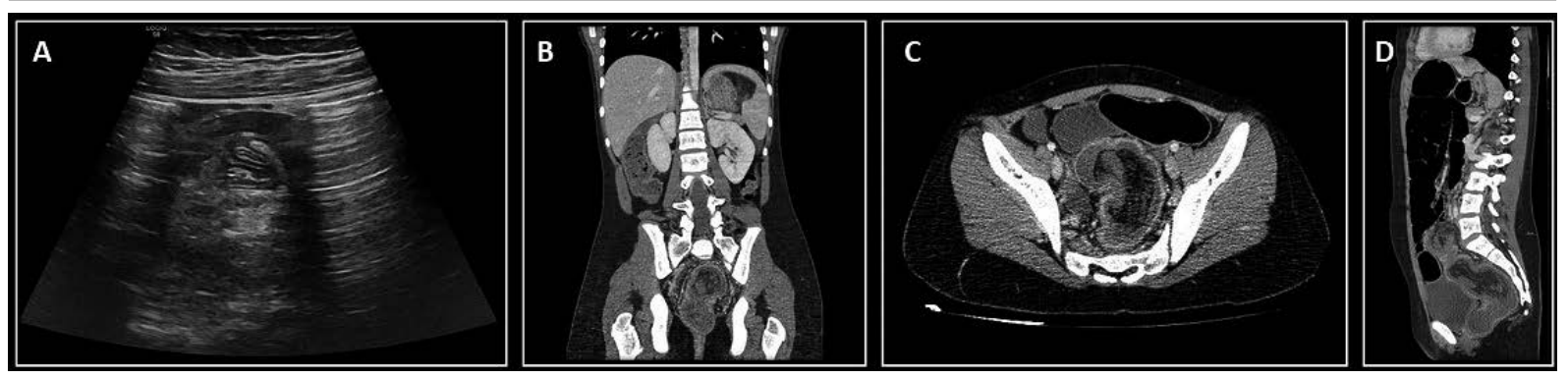

A. Ecografía abdominal en la que se observa una lesión ecogénica en el hipogastrio sugestiva de invaginación sigmoidea con vascularización preservada.

B, C, D. Corte coronal, transversal y sagital de la tomografía computada, en la que se visualiza una imagen compatible con invaginación sigmoidea con un nódulo hipovascular de $40 \mathrm{~mm}$ en el extremo distal.
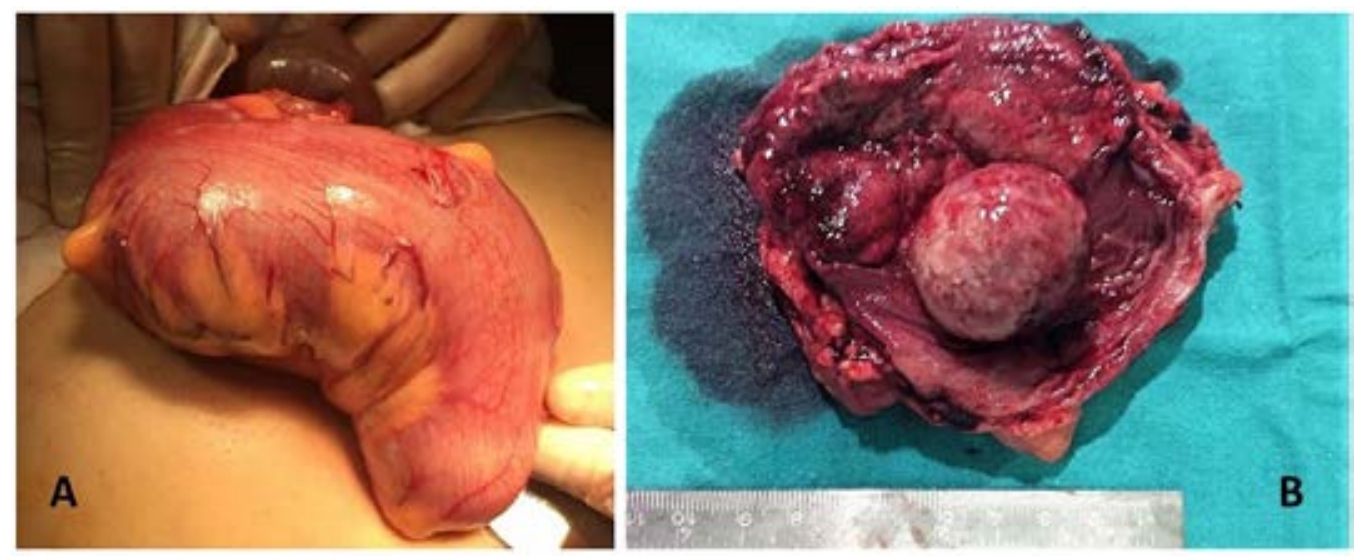

A. Imagen de invaginación sigmoidea de aproximadamente $15 \mathrm{~cm}$.

B. Tumoración redondeada intraluminal, grisácea, de $4 \times 3 \times 3 \mathrm{~cm}$, con ulceración de la mucosa, compatible con schwannoma de colon. 
ocasional de empalizadas nucleares. El análisis inmunohistoquímico mostró que las células neoplásicas expresaban S-100, vimentina y CD34, que fueron negativos para actina de músculo liso, desmina, DOG1, CKIT, Ckae1/ae3 y B-catenina (Figura 3). El índice proliferativo Ki-67 fue bajo (1\%). El índice mitótico no mostró potencial maligno. Por lo tanto, la anatomía patológica confirmó el diagnóstico de schwannoma de colon sigmoide.

Se realizó el estudio genético, que descartó neurofibromatosis y schwannomatosis.

\section{DISCUSIÓN}

Verocay describió por primera vez los schwannomas en $1910 .{ }^{3}$ Desde entonces, los casos de tumores mesenquimales han ido en aumento, debido al desarrollo de las técnicas inmunohistoquímicas de tinción. ${ }^{3}$ Sin embargo, los schwannomas primarios de colon y recto no asociados a neurofibromatosis sistémica son aún extremadamente raros. Por lo general, los schwannomas son tumores benignos de origen ectodérmico de crecimiento lento que pueden degenerar a tumores malignos (sarcomas) en raras ocasiones. ${ }^{1,3}$ Sin embargo, está descrita la capacidad de degeneración maligna en aquellos casos que no se extirpan. ${ }^{3,5}$ Estos tumores son, generalmente, asintomáticos, pero pueden producir síntomas inespecíficos, como dolor, fatiga y fiebre, y síntomas gastrointestinales, como tenesmo rectal, dolor abdominal, rectorragia, alteraciones deposicionales u obstrucción de colon. ${ }^{1,5}$ La presentación clínica como invaginación es extremadamente rara.
Solo existe un caso descrito en la literatura de una invaginación intestinal secundaria a un schwannoma de sigma, como nuestro caso. ${ }^{2}$ A pesar de que la invaginación intestinal es frecuente en los niños menores de 2 años, es poco habitual en los niños mayores, $\mathrm{y}$, además, la invaginación colocolónica es extremadamente infrecuente $(<5 \%$ de todas las invaginaciones en la edad pediátrica) y son, por lo general, causadas por otras patologías que funcionan como cabeza de invaginación, ${ }^{7}$ como divertículo de Meckel, duplicaciones intestinales, linfomas, hiperplasia nodular linfoidea o malformaciones vasculares.

Las pruebas de imagen son inespecíficas y el diagnóstico del schwannoma intestinal es difícil. El diagnóstico diferencial debe realizarse, principalmente, con los tumores estromales gastrointestinales (gastrointestinal stromal tumor; GIST, por sus siglas en inglés), debido a su semejanza macroscópica. ${ }^{8}$ Ambos tipos de tumores están localizados en la pared del tracto gastrointestinal y muestran un patrón de crecimiento similar. ${ }^{8,9}$ La TAC muestra una masa intramural homogénea que protruye de la mucosa colónica, lo que se diferencia de los GIST, que son masas heterogéneas. No suele haber evidencia de cápsula tumoral, cambios quísticos, formación de cavidades, necrosis o calcificaciones, características que se describen con más frecuencia en los GIST. ${ }^{9}$ Generalmente, las pruebas de imagen no confirman el diagnóstico de este tipo de tumores, por lo que es necesaria la biopsia o resección completa, como en nuestro caso, dada la agudeza del cuadro, para alcanzar un diagnóstico definitivo. ${ }^{8}$
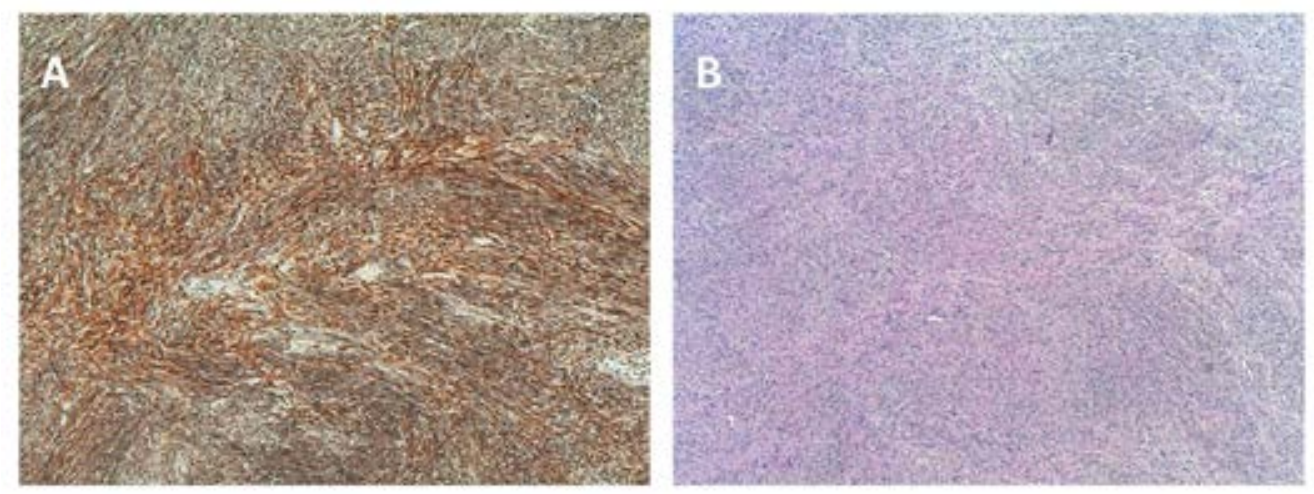

A. Inmunorreactividad frente a la proteína S-100.

B. Células fusiformes sin atipia en haces entrecruzados. 
Macroscópicamente, los schwannomas intestinales suelen presentarse como pólipos bien definidos, lobulados, blanco-amarillentos que pueden ulcerar la mucosa, como se puede observar en la Figura 2, y, en raras ocasiones, pueden ser de consistencia dura, sólidos, ulcerados o calcificados. ${ }^{1,5}$ Microscópicamente, se disponen en un patrón de haces entrelazados, rodeados de un infiltrado de células linfoides con centros germinales y pequeños focos de atipia celular con escasa actividad mitótica. ${ }^{1}$ En el sistema gastrointestinal, se originan con mayor frecuencia del plexo de Auerbach que del plexo de Meissner. ${ }^{5}$ El análisis inmunohistoquímico de las células tumorales se considera la herramienta diagnóstica óptima para este tipo de tumor y es muy útil para el diagnóstico diferencial con otras lesiones, como GIST, tumores autonómicos gastrointestinales (gastrointestinal autonomic nerve tumors; GANT, por sus siglas en inglés), leiomiomas o leiomiosarcomas, que pueden presentar un comportamiento más agresivo. Los schwannomas, generalmente, presentan reactividad postiva para S-100, vimentina y la proteína ácida fibrilar gliar (glial fibrillary acidic protein; GFAP, por sus siglas en inglés), y no presentan reactividad para el receptor transmembrana de la tirosin-kinasa, denominado c-KIT o CD117, CD34, actina o citoqueratinas, que aparecen de forma típica en los tumores GIST, GANT o tumores musculares. ${ }^{10} \mathrm{El}$ índice proliferativo Ki-67 se recomienda como indicador de malignidad. Se considera que su positividad $(\geq 5 \%)$ está estrictamente relacionada con una mayor agresividad tumoral y más del $10 \%$ se consideran malignos. ${ }^{5}$ En nuestro caso, el índice proliferativo Ki-67 fue del $1 \%$. Tras el diagnóstico, la opción terapéutica de elección se considera la resección radical con márgenes libres, debido a su tendencia de recurrencia local o malignización en aquellos casos que no se tratan. ${ }^{2,3}$ El abordaje quirúrgico dependerá del tamaño del tumor, localización y el patrón histopatológico, en aquellos casos en los que se pueda realizar una biopsia previa para establecer el diagnóstico anatomopatológico. El pronóstico para estos pacientes es bueno, ya que la recurrencia o la presencia de metástasis son raras.

En conclusión, se presenta un caso clínico extremadamente raro en una niña, tanto por la presencia de un schwannoma sigmoideo como por su presentación clínica como invaginación intestinal de sigma. A pesar de lo poco frecuente que es el schwannoma de colon, se deben conocer sus posibles presentaciones clínicas, características y tratamiento, y tenerlo en cuenta en el diagnóstico diferencial de tumoraciones localizadas en el colon.

\section{REFERENCIAS}

1. Verdú-Fernández MA, Guillén-Paredes MP, GarcíaGarcía ML, García-Marín JA, et al. Schwannoma en colon descendente: presentación de una neoplasia en rara localización. Rev Esp Enferm Dig. 2013; 105(8):504-5.

2. Petrie BA, Ho JM, Tolan AM. Schwannoma of the sigmoid colon: a rare cause of sigmoidorectal intussusception. ANZ J Surg. 2014; 84(12):990-1.

3. Wang WB, Chen WB, Lin JJ, Xu JH, et al. Schwannoma of the colon: A case report and review of the literature.Oncol Lett. 2016; 11(4):2580-2.

4. Kanneganti K, Patel H, Niazi M, Kumbum K, et al. Cecal Schwannoma: A rare cause of gastrointestinal bleeding in a young woman with review of literature. Gastroenterol Res Pract. 2011; 2011:142781.

5. Çakır T, Aslaner A, Yaz M, Gündüz U. Schwannoma of the sigmoid colon. BMJ Case Rep. 2015; 2015:bcr2014208934.

6. Zippi M, Pica R, Scialpi R, Cassieri C, et al. Schwannoma of the rectum: A case report and literature review. World J Clin Cases. 2013; 1(1):49-51.

7. Soccorso G, Puls F, Richards C, Pringle H, et al. A ganglioneuroma of the sigmoid colon presenting as leading point of intussusception in a child: a case report. J Ped Surg. 2009; 44(1):17-20.

8. Dickson-Lowe RA, James CL, Bailey CMH, Abdulaal Y. Caecal schwannoma: a rare cause of per rectal bleeding in a 72-year-old man. BMJCase Rep. 2014; 2014:bcr2014205643.

9. Levy AD, Quiles AM, Miettinen M, Sobin LH. Gastrointestinal schwannomas: CT features with clinicopathologic correlation. AJR Am J Roentgenol. 2005; 184:797-802.

10. Hou YY, Tan YS, Xu JF, Wang XN, et al. Schwannoma of the gastrointestinal tract: A clinicopathological, immunohistochemical and ultrastructural study of 33 cases. Histopathology. 2006; 48(5):536-45. 